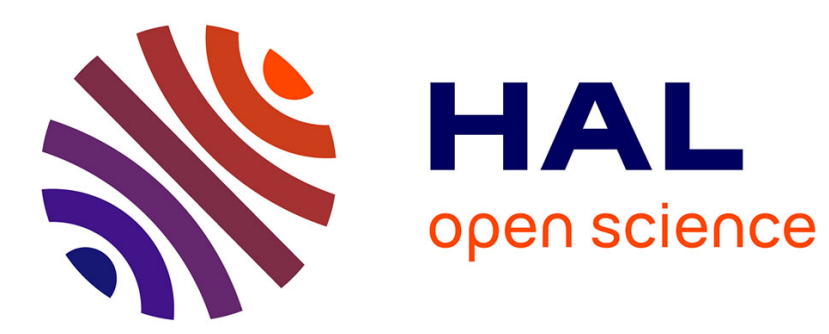

\title{
Pure emitter dephasing: A resource for advanced solid-state single-photon sources
}

\author{
Alexia Auffèves, Jean-Michel Gérard, Jean-Philippe Poizat
}

\section{To cite this version:}

Alexia Auffèves, Jean-Michel Gérard, Jean-Philippe Poizat. Pure emitter dephasing: A resource for advanced solid-state single-photon sources. Physical Review A : Atomic, molecular, and optical physics [1990-2015], 2009, 79, pp.053838. 10.1103/PhysRevA.79.053838 . hal-00999555

\section{HAL Id: hal-00999555 https://hal.science/hal-00999555}

Submitted on 3 Jun 2014

HAL is a multi-disciplinary open access archive for the deposit and dissemination of scientific research documents, whether they are published or not. The documents may come from teaching and research institutions in France or abroad, or from public or private research centers.
L'archive ouverte pluridisciplinaire HAL, est destinée au dépôt et à la diffusion de documents scientifiques de niveau recherche, publiés ou non, émanant des établissements d'enseignement et de recherche français ou étrangers, des laboratoires publics ou privés. 


\title{
Pure emitter dephasing: A resource for advanced solid-state single-photon sources
}

\author{
Alexia Auffèves, ${ }^{1}$ Jean-Michel Gérard, ${ }^{2}$ and Jean-Philippe Poizat ${ }^{1}$ \\ ${ }^{1}$ CEA/CNRS/UJF Joint Team "Nanophysics and Semiconductors," Institut Néel, CNRS, \\ BP 166, 25 rue des Martyrs, 38042 Grenoble Cedex 9, France \\ ${ }^{2}$ CEA/CNRS/UJF Joint Team "Nanophysics and Semiconductors," CEA/INAN/SP2M, \\ 17 rue des Martyrs, 38054 Grenoble, France \\ (Received 6 August 2008; published 19 May 2009)
}

\begin{abstract}
We have computed the spectrum emitted spontaneously by a quantum dot coupled to an arbitrarily detuned single mode cavity, taking into account pure dephasing processes. We show that if the emitter is incoherent, the cavity can efficiently emit photons with its own spectral characteristics. This effect opens unique opportunities for the development of devices exploiting both cavity quantum electrodynamic effects and pure dephasing, such as wavelength-stabilized single-photon sources robust against spectral diffusion.
\end{abstract}

DOI: 10.1103/PhysRevA.79.053838

PACS number(s): 42.50.Pq, 42.50.Ct, 42.50.Gy, 42.65.Hw

Experiments previously restricted to the field of atomic physics with isolated two-level atoms and high- $Q$ cavities can nowadays be implemented using solid-state emitters and optical microcavities, paving the road toward integrable information processing. The strong confinement of electron and holes in semiconductor quantum dots (QDs) results in a discretization of their electronic energy levels and to an attractive set of atomlike properties, such as spectrally narrow emission lines at low temperature, and the ability to generate quantum states of light, such as single photons [1,2]. At the same time, impressive progress in the technology of solidstate cavities allows us to observe cavity quantum electrodynamic effects for a single QD embedded in a solid-state optical microcavity, such as Purcell effect [2] and vacuum Rabi splitting [3-8]. Nevertheless, a QD is far from behaving like an isolated atom. In particular, it interacts with the phonons of the matrix it is embedded in, giving rise to sidebands in addition to the so-called zero-phonon line (ZPL) [9]. At sufficiently low temperature yet, the emission in the ZPL remains predominant $[10,11]$, allowing to model these systems as effective two-level systems. Another difference is due to the random trapping of carriers in the vicinity of the QD, leading to the observation of spectral jumps at long time scale [12], and at shorter time scales, to the broadening of the homogeneous linewidth of the transition [13]. This additional source of decoherence can be attributed to the measurement by the environment of the state of the QD, resulting in a loss of indistinguishability of the emitted photons [14]. The best degree of indistinguishability reported to date for semiconducting QDs, which has been obtained for single InAs QDs in pillar microcavities, is on the order of $80 \%[15,16]$.

For this very reason, pure dephasing mechanisms are often considered as a drawback, likely to reduce severely the potential interest of QDs for quantum information processing and communication (QIPC). In this paper, we show that pure dephasing can by contrast also be seen as a novel resource for QIPC that is specific to solid-state emitters. It has been pointed out recently that pure emitter dephasing has a crucial influence on the shape of the emission spectra of QD-cavity systems [17-19], and leads to a strong increase in the emission at the cavity energy for detuned systems. Moreover, pure dephasing rate is experimentally controllable by tuning the temperature of the setup, the pumping rate [20], and the electrical field in the vicinity of the QD [21]. Pure dephasing provides thus a supplementary degree of freedom, specific to QDs, which, combined with cavity quantum electrodynamic effects, offers appealing potentialities to develop advanced solid-state single-photon sources.

In this paper, we compute and analyze the spectrum $S_{\text {cav }}$ spontaneously emitted by a cavity coupled to a QD initially fed with a single exciton, and the probability $P_{c a v}$ for the quantum of energy to be emitted in the cavity channel of losses. Like in [22], the spectrum $S_{c a v}$ is normalized with respect to the frequency $\omega$. The quantities $S_{\text {cav }}$ and $P_{c a v}$ can be measured by a frequency-resolved detector placed in the radiation pattern of the cavity mode. This generalizes the study held in [22], where the only considered emitter was an isolated atom, undergoing no pure dephasing. The system is pictured in Fig. 1(a). The QD's and the cavity's frequencies are denoted $\omega_{0}$ and $\omega_{\text {cav }}$, the QD-cavity detuning being $\delta$ $=\omega_{\text {cav }}-\omega_{0}$. The annihilation operator in the cavity mode is $a$ and the atomic operators $\sigma_{-}=|g\rangle\langle e|$ and $\sigma_{+}=|e\rangle\langle g|$, where $|e\rangle$ and $|g\rangle$ are the upper and ground states of the QD, respectively. In addition to the cavity mode, the QD is coupled to a continuum of leaky modes. The spontaneous emission rate in this lossy channel is denoted $\gamma$. This rate is usually measured in time-resolved experiments, the QD and the cavity being strongly detuned, and ranges from 1 to $10 \mu \mathrm{eV}$. Pure dephasing is considered in an effective manner by an additional relaxation term $\gamma^{*}$ in the evolution equation of the atomic coherence. If the QD is not coupled to the cavity mode, the emission line shape remains Lorentzian, its width being $\gamma+\gamma^{*}$. This intrinsic spectrum is further denoted $S_{Q D}^{0}$. From an experimental point of view, this modeling is well

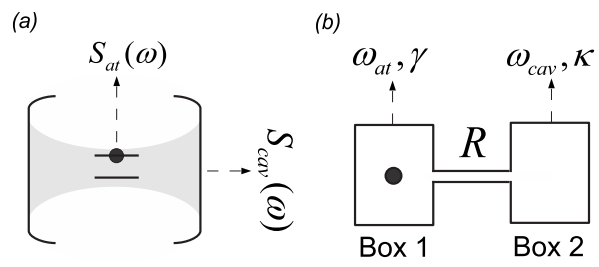

FIG. 1. (a) System under study. (b) Equivalent system in the incoherent-emitter regime: two connected boxes exchanging a quantum of energy. 

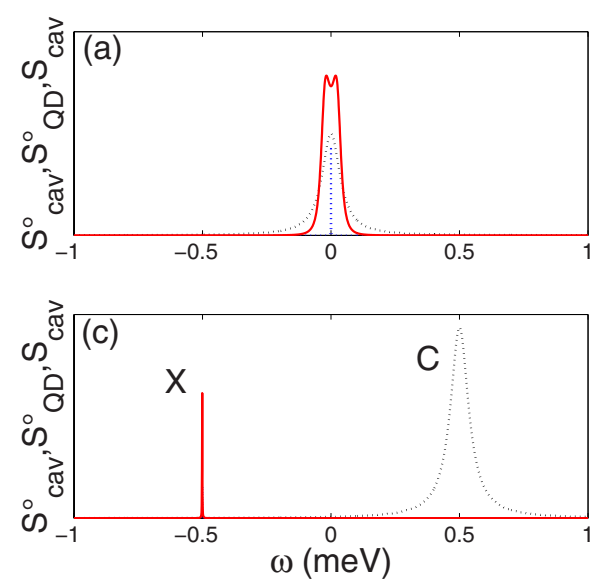

adapted to the so-called motional-narrowing regime, where Lorentzian emission lines are observable [20,21,23]. Pure dephasing rate in this regime can be increased up to a few hundreds of $\mu \mathrm{eV}$ [20]. On the other hand, the cavity's losses induce a finite linewidth $\kappa$ of the mode, which typically scales like $\kappa=100 \mu \mathrm{eV}[6,8]$. We denote $S_{\text {cav }}^{0}$ the empty cavity spectrum as it could be registered in a transmission experiment. Finally, the QD-cavity coupling strength is denoted $g$, and checks for the best couplings $g \sim 100-200 \mu \mathrm{eV}$ $[3,8]$. The system is initially prepared in the state $|e, 0\rangle$. Such an initial state can be implemented by quasiresonantly pumping the QD as in Refs. [6,7], so that the QD is properly modeled by a two-level system. The evolution of the system is described by a master equation, and remains restricted in the subspace spanned by the basis $\{|e, 0\rangle,|g, 1\rangle,|g, 0\rangle\}$. The populations' evolution follows the equations

$$
\begin{gathered}
\frac{d\left\langle a^{\dagger} a\right\rangle}{d t}=-\kappa\left\langle a^{\dagger} a\right\rangle+g\left\langle\sigma_{+} a\right\rangle+g\left\langle a^{\dagger} \sigma_{-}\right\rangle, \\
\frac{d\left\langle\sigma_{+} \sigma_{-}\right\rangle}{d t}=-\gamma\left\langle\sigma_{+} \sigma_{-}\right\rangle-g\left\langle\sigma_{+} a\right\rangle-g\left\langle a^{\dagger} \sigma_{-}\right\rangle, \\
\frac{d\left\langle\sigma_{+} a\right\rangle}{d t}=-i \delta\left\langle\sigma_{+} a\right\rangle-\frac{\gamma+\gamma^{*}+\kappa}{2}\left\langle\sigma_{+} a\right\rangle+g\left(\left\langle\sigma_{+} \sigma_{-}\right\rangle-\left\langle a^{\dagger} a\right\rangle\right) .
\end{gathered}
$$

We have computed the spectrum $S_{\text {cav }}$ emitted by the cavity using the formula of Glauber [24]. It fulfills

$$
S_{c a v} \propto \frac{1}{\left|\omega-\lambda_{+}\right|^{2}} \frac{1}{\left|\omega-\lambda_{-}\right|^{2}},
$$

where $\lambda_{+}$and $\lambda_{-}$are the roots of the secular equation

$$
\left(\tilde{\omega}_{a t}-\omega\right)\left(\tilde{\omega}_{c a v}-\omega\right)-g^{2}=0 .
$$

We have introduced the complex frequencies of the QD and of the cavity in the absence of coupling, $\widetilde{\omega}_{0}=\omega_{0}-i(\gamma$ $\left.+\gamma^{*}\right) / 2$ and $\widetilde{\omega}_{c a v}=\omega_{c a v}-i \kappa / 2$. If the coupling is weak with respect to the detuning or to the QD's and cavity's widths, the roots $\lambda_{+}$and $\lambda_{-}$equal the intrinsic frequencies $\widetilde{\omega}_{0}$ and $\widetilde{\omega}_{\text {cav }}$, and the spectrum emitted by the cavity simply writes as
FIG. 2. (Color online) Spectrum $S_{\text {cav }}$ emitted by the coupled cavity (red solid line) and spectra emitted by the QD, $S_{Q D}^{0}$ (blue dotted line), and by the cavity, $S_{\text {cav }}^{0}$ (black dashed line), if they were uncoupled for $g=35 \mu \mathrm{eV}, \kappa$ $=85 \mu \mathrm{eV}$, and $\gamma=1 \mu \mathrm{eV}[(\mathrm{a})$ and (b)] in the resonant case and [(c) and (d)] in the case $\delta=1 \mathrm{meV}$. $[(\mathrm{a})$ and $(\mathrm{c})] \gamma^{*}=0 ;[(\mathrm{b})$ and (d) $]$ $\gamma^{*}=0.5 \mathrm{meV}$.

showing that the cavity behaves as a spectral filter for the light emitted by the QD. As a consequence, if the QD's emission line is narrower than the cavity linewidth $\left(\gamma+\gamma^{*}<\kappa\right)$, photons are mostly emitted at the QD's frequency, and at the cavity's frequency in the opposite case as it appears in Fig. 2. We took the parameters of Ref. [6], and considered the evolution of the spectrum emitted by the cavity when, starting from $\delta=0$ [Figs. 2(a) and 2(b)], one switches to $\delta=1 \mathrm{meV}$ $\sim 11 \kappa$ [Figs. 2(c) and 2(d)]. This quantity is the typical amplitude of spectral jumps as they can be observed for a single nanocrystal coupled to a microsphere [12]. It also provides an upper bound for the range of spectral diffusion affecting the emission line of self-assembled QDs [10,23]. First we consider the case when $\gamma^{*} \sim 0$ which corresponds to the experimental conditions of Ref. [6]. At resonance one can almost observe the vacuum Rabi doublet [Fig. 2(a)]. If the excitonic transition's frequency changes, the cavity emits photons at this new frequency [Fig. 2(c)]. On the contrary, if $\gamma^{*}$ is increased so that it overcomes the cavity linewidth $\kappa$ (we took $\gamma^{*}=0.5 \mathrm{meV}$, which corresponds to reasonable experimental parameters), photons are emitted at the cavity frequency [Fig. 2(d)]. We stress that in the regime of parameters we consider, the spectrum $S_{c a v}$ of the photons emitted by the detuned cavity exactly matches the spectrum $S_{c a v}^{0}$ of the empty cavity mode. This is because the QD's spectrum $S_{Q D}^{0}$ is flat in the vicinity of the cavity's frequency [Eq. (4)]: the QD behaves as an internal white-light source. Interestingly, increasing the pure dephasing rate provides a safe way to measure the intrinsic quality factor of a solid-state cavity, even though it is only fed with a single emitter. This mechanism may also allow enforcement of a single-photon source to emit photons at the cavity's frequency, making it robust against spectral jumps and spectral diffusion. We emphasize that the figures are plotted with realistic experimental parameters, showing that this functionality can be achieved with state-of-the-art QDs and cavities, provided one manages to control the environment of the QD.

Until now we have only paid attention to the normalized spectrum $S_{\text {cav }}$ emitted by the cavity mode. In the framework of photonic devices it is also essential to estimate the efficiency $P_{c a v}$ of the emission process. First note that if pure 

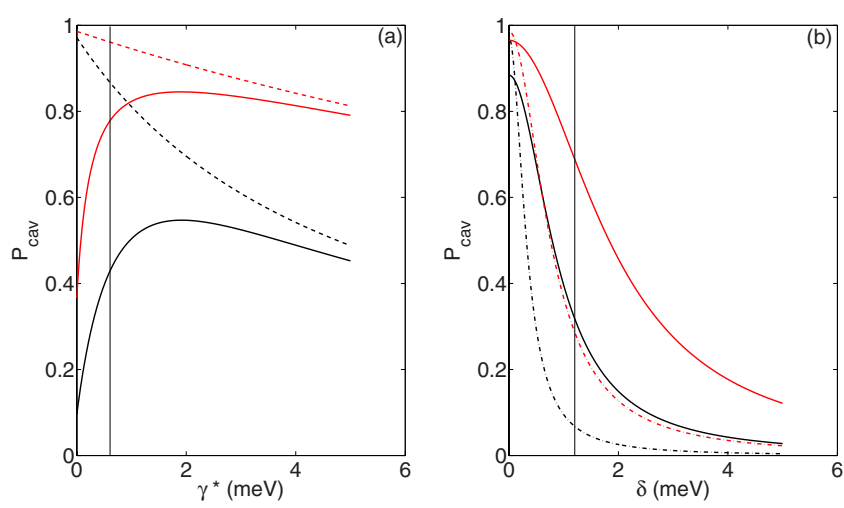

FIG. 3. (Color online) Efficiency $P_{\text {cav }}$ as a function of (a) the pure dephasing rate $\gamma^{*}(\mathrm{~b})$ the QD-cavity detuning $\delta$. Black plots: $g=35 \mu \mathrm{eV}, \kappa=85 \mu \mathrm{eV}$, and $\gamma=1 \mu \mathrm{eV}$ (parameters of Ref. [6] and Fig. 2). Red plots: $g=76 \mu \mathrm{eV}, \kappa=100 \mu \mathrm{eV}$, and $\gamma=1 \mu \mathrm{eV}$ (parameters of Ref. [8]). (a) Dashed line: $\delta=0$; solid line: $\delta$ $=1 \mathrm{meV}$. (b) Dash-dotted line: $\gamma^{*}=0$; solid line: $\gamma^{*}=0.5 \mathrm{meV}$.

dephasing is sufficiently strong, coherences can be adiabatically eliminated in set of equations (1). The dynamics of the system is now described by classical probabilities and corresponds to the diffusion of a particle between an atomic box and a cavity box, each box having a probability $\gamma$ (respectively, $\kappa$ ) per unit of time to lose the particle as it is pictured in Fig. 1(b). The coupling rate $R$ between the two boxes fulfills

$$
R\left(\gamma^{*}, \delta\right)=\frac{4 g^{2}}{\kappa+\gamma+\gamma^{*}} \frac{1}{1+\left(\frac{2 \delta}{\kappa+\gamma+\gamma^{*}}\right)^{2}},
$$

and evolves like the overlap between the uncoupled QD and cavity spectra $S_{O D}^{0}$ and $S_{c a v}^{0}$. Using this picture the efficiency $P_{c a v}$ can easily be computed. One finds

$$
P_{c a v}=\frac{\kappa}{\kappa+\gamma} \frac{\mathcal{C}}{1+\mathcal{C}}
$$

where we have introduced the cooperativity $\mathcal{C}$, fulfilling

$$
\mathcal{C}=R\left(\frac{1}{\kappa}+\frac{1}{\gamma}\right) .
$$

Note that although these expressions are most easily derived in the incoherent regime, they are in fact valid for all values of the pure dephasing rate $\gamma^{*}$. In particular, if $\gamma^{*}=0$, one recovers the form computed in Ref. [17] in the resonant case and in the absence of pure dephasing.

We have represented in Fig. 3(a) the evolution of the efficiency $P_{c a v}$ of the cavity emission process as a function of the pure dephasing rate, in the resonant case and in the case where the QD and the cavity are detuned by $\delta=1 \mathrm{meV}$. We considered the parameters of Refs. [6,8]. In the resonant case, the efficiency drops because the overlap between the uncoupled spectra $S_{Q D}^{0}$ and $S_{c a v}^{0}$ decreases, and thus the cooperativity $\mathcal{C}$ of the corresponding single-photon source as mentioned above. On the contrary, when the QD and the cavity are detuned, the overlap starts to increase with respect to the pure dephasing rate, so does the efficiency of the process as it appears in the figure. The fact that pure dephasing enhances the effective coupling between a QD and a detuned cavity mode may provide a partial explanation for the lasing observed for a photonic crystal cavity mode coupled to a few detuned quantum dots [25], as suggested in [18]. The optimum is reached for $\kappa+\gamma^{*}=2 \delta$, and the maximal cooperativity equals $g^{2} / \gamma \delta$. The enhancement that could be reached in the case of Ref. [8] is more spectacular, as the coupling strength $g$ is bigger than in Ref. [6].

The evolution of the efficiency $P_{c a v}$ as a function of the detuning $\delta$ is plotted in Fig. 3(b), for $\gamma^{*}=0$ and $\gamma^{*}$ $=0.5 \mathrm{meV}$. It appears that if $\delta \leq \kappa$, a single-photon source undergoing pure dephasing is more efficient than the same source perfectly isolated from its environment. This effect could be exploited to increase the fabrication yield of efficient single-photon sources prepared with a sample of inhomogeneously broadened QDs. Another advantage of pure dephasing is that it makes the efficiency of a single-photon source less sensitive to spectral jumps or spectral diffusion. As an example, one considers how the efficiency of a singlephoton source is affected if the QD and the cavity, initially on resonance, are detuned by $\delta=1 \mathrm{meV}$. For the parameters of Ref. [6], without pure dephasing, the efficiency of the device drops from $97 \%$ to $10 \%$, whereas it only jumps from $90 \%$ to $40 \%$ in the presence of pure decoherence. With the parameters of Ref. [8], the efficiency drops from $99 \%$ to $37 \%$ without pure dephasing, whereas with pure dephasing, it only drops from $97 \%$ to $76 \%$. As a consequence, pure dephasing allows stabilization not only of the frequency of the device, but also its efficiency.

At this stage, one can highlight a major difference between a QD and an isolated atom. Cavity filtering requires at least the condition $\gamma+\gamma^{*}>\kappa$. On the other hand, a necessary condition to have efficient cavity emission is $\kappa>\gamma$. It is obvious that the two conditions cannot match if $\gamma^{*}=0$ : as a consequence, there may be no efficient cavity filtering for an isolated atom. Pure dephasing appears as a supplementary degree of freedom, specific to QDs, allowing the engineering of their emission linewidth and their losses in a decoupled way. As a consequence, it is possible to combine low atomic losses and cavity filtering to achieve an efficient wavelengthstabilized single-photon source. For example, in the case of Ref. [8], with $\delta=1 \mathrm{meV}$, the filtering condition is fulfilled with $\gamma^{*}=0.5 \mathrm{meV}$, and the efficiency of the process is $76 \%$ as mentioned above. Moreover, the process is all the more efficient than the QD's losses are reduced. Namely, very high values for $P_{c a v}$ can be reached, provided $\gamma \ll \kappa$ and $R\left(\gamma^{*}, \delta\right)$ [see Eq. (7)]. This is intuitive, as the quantum has no other option apart from being released in the cavity channel of losses: the ideal single-photon source is nothing but a shielded atomic box connected to a lossy cavity. Considering again the case of Ref. [8], the local density of leaky modes could be engineered to reduce the corresponding spontaneous emission rate to $\gamma=0.1 \mu \mathrm{eV}$. In this case, the efficiency reaches $96 \%$. With these parameters, most photons are spontaneously emitted at the cavity frequency, even though the cavity is detuned from the QD, providing efficient energy conversion. 
To conclude, it is nice to notice that the emitter's decoherence can also be exploited to develop a source of indistinguishable photons. Indistinguishable photons are resources in the frame of quantum computation with linear optics [26]. A necessary condition of indistinguishability is that photons are Fourier-transform limited, namely, that the spectral width $\delta \nu$ of the photonic peak corresponds to the inverse of its duration $\tau$. Like it appears in Eq. (2), a cavity coupled to a QD emits photons whose frequency and linewidth are imposed by the narrowest root of Eq. (3). In the usual approach, pure dephasing is very weak, and the linewidth of the emitted photons corresponds to the linewidth of the QD "dressed" by the cavity, namely, in the resonant case, $\delta \nu=\gamma+\gamma^{*}+4 g^{2} / \kappa$. The duration $\tau$ of the wave packet checks $\tau^{-1}=\gamma+\kappa / 4 g^{2}$, which corresponds to the relaxation of the QD in the cavity in the Purcell regime. To recover indistinguishability, the usual strategy consists of reducing $\gamma^{*}$ by lowering the temperature and quasiresonantly pumping the system, and by increasing the relaxation rate by Purcell effect $[15,16]$. Nevertheless, this strategy has its limits as it appears in Ref. [14]. In particular, the relaxation time must remain large enough so that the process remains insensitive to the jitter due to the optical pumping time. Moreover, as the spectral characteristics of the emitted photons are governed by the emitter, one is linearly sensitive to any variation in the frequency or in the emission linewidth of the QD.

On the contrary, an alternative strategy consists of increasing the pure dephasing rate, so that it overcomes the cavity linewidth. Provided the QD's spectrum is sufficiently flat in the vicinity of the cavity's frequency (condition of white-light regime), the frequency and the width of the emitted photons are imposed by the cavity as explained above. Moreover, it can easily be shown that the lifetime $\tau$ of the quantum in the atom-cavity system checks

$$
\tau^{-1}=\frac{\kappa+\gamma}{2}+R-\sqrt{\left(\frac{\kappa-\gamma}{2}\right)^{2}+R^{2}} .
$$

If $\gamma=\kappa$, this lifetime equals the cavity lifetime, and the emitted photons are Fourier-transform limited, and thus indistinguishable. In this approach, the cavity is used as an integrated spectral filter that restores the temporal indistinguishability of the emitted photons, initially degraded by the interaction of the QD with its environment [14]. It may provide solid-state physicists with an original method to generate photons showing a degree of indistinguishability as high as desired, and relaxes the constraint on low temperature. Nevertheless, this method is intrinsically limited. The whitelight regime is reached indeed when there is a poor overlap between the QD's and cavity's uncoupled spectra, and as a consequence, a poor cooperativity $\mathcal{C}$. Thus there is a trade-off between the degree of indistinguishability of the emitted photons and the efficiency of the source, which is inherent to any method based on spectral filtering. To have a glimpse of the performances of the device, we have considered the wavelength-stabilized single-photon source studied above, supposing now that $\kappa=\gamma=10 \mu \mathrm{eV}$. In practice, the fabrication of such a device should be doable in the near future, as nowadays, state-of-the-art QD and cavities already allow achievement of $\kappa=10 \gamma[3-6,8]$, and impressive progress has been recently witnessed for semiconductor-based cavities such as micropillars [27], microdisks [28], or photonic crystal cavities [29]. First we have computed the degree of indistinguishability $d=\tau / \delta \nu$ of the emitted photon in the resonant case, which checks $d=80 \%$. A convenient way to reach the white-light regime is to strongly detune the QD from the cavity mode. For a detuning $\delta=1.5 \mathrm{meV}=150 \kappa$, the efficiency of the source is $3 \%$, whereas a degree of indistinguishability $d=97 \%$ is reached. The trade-off between efficiency and indistinguishability clearly appears here, as this method only consists of spectral filtering "on chip." Nevertheless, it paves the road toward the generation of indistinguishable photons at finite temperature.

As underlined in Refs. [18,19], pure dephasing could explain part of the results obtained for the emission of a semiconducting cavity coupled to a detuned QD $[6,8]$. Providing a clear understanding of these striking properties is very challenging. All explanations, including ours, involve the broadening of the QD's emission line, combined with cavity filtering. Within our model, the QD remains properly described by a two-level system. In other explanations, the broadening of the QD's emission line is due to a continuum of final states for the excitonic transition: namely, Press et al. [6] invoked phonon-assisted processes, and Kaniber et al. [30], a continuum of final charged states of the dot. The next step will be to take into account the cavity, to quantitatively estimate the efficiency of each relaxation channel. Note that within the framework of these three models, the correlation measurements achieved on the cavity mode should be antibunched, which is not the case in Ref. [8]. As underlined in [19], the QD is nonresonantly pumped in this reference, which may induce multiphotonic processes. As it is stated in the beginning, our model is better adapted to the experiments where the QD is properly described by a two-level atom, which is the case if it is quasiresonantly pumped as in [6]. Let us stress that resonant excitation like that in [7] seems to further reduce multiphoton processes. This excitation regime, which avoids jitter and spectral fluctuation, is quite well described by the model exposed in this paper.

We have shown that pure decoherence is a degree of freedom specific to solid-state emitters, giving rise to unexpected regimes for cavity quantum electrodynamics. Provided one succeeds in controlling their environment, quantum dots could in the near future be used as toy models to explore these new regimes, making them free from the two-level atom paradigm [3-5,31]. It also appears that pure dephasing, far from being a drawback, is a resource that can be exploited to develop advanced nanophotonic devices such as frequency-stabilized solid-state single-photon sources, opening promising perspective for quantum computation on chip.

A.A. thanks Marcelo França Santos, Xavier Letartre, Pierre Viktorovitch, and Christian Seassal for all the conversations. The authors thank J. Moerk for pointing to their attention the crucial role of emitter dephasing on QD-cavity spectra prior to the publication of [18]. J.-M.G. acknowledges partial support from the IST-FET QPhoton project. This work was supported by IP European project QAP (Contract No. 15848). 
[1] C. Santori, M. Pelton, G. Solomon, Y. Dale, and Y. Yamamoto, Phys. Rev. Lett. 86, 1502 (2001).

[2] E. Moreau et al., Appl. Phys. Lett. 79, 2865 (2001).

[3] E. Peter, P. Senellart, D. Martrou, A. Lemaitre, J. Hours, J. M. Gerard, and J. Bloch, Phys. Rev. Lett. 95, 067401 (2005).

[4] J. P. Reithmaier et al., Nature (London) 432, 197 (2004).

[5] T. Yoshie et al., Nature (London) 432, 200 (2004).

[6] D. Press, S. Gotzinger, S. Reitzenstein, C. Hofmann, A. Loffler, M. Kamp, A. Forchel, and Y. Yamamoto, Phys. Rev. Lett. 98, 117402 (2007).

[7] D. Englund, A. Majumdar, A. Faraon, M. Toishi, N. Stoltz, P. Petroff, and J. Vuckovic, e-print arXiv:0902.2428.

[8] K. Hennessy et al., Nature (London) 445, 896 (2007).

[9] B. Krummheuer, V. M. Axt, and T. Kuhn, Phys. Rev. B 65, 195313 (2002).

[10] I. Favero, G. Cassabois, R. Ferreira, D. Darson, C. Voisin, J. Tignon, C. Delalande, G. Bastard, P. Roussignol, and J. M. Gerard, Phys. Rev. B 68, 233301 (2003).

[11] L. Besombes, K. Kheng, L. Marsal, and H. Mariette, Phys. Rev. B 63, 155307 (2001).

[12] N. Le Thomas, U. Woggon, O. Schöps, M. V. Artemyev, M. Kazes, and U. Banin, Nano Lett. 6, 557 (2006).

[13] L. Coolen, X. Brokmann, P. Spinicelli, and J. P. Hermier, Phys. Rev. Lett. 100, 027403 (2008).

[14] A. Kiraz, M. Atature, and A. Immamoglu, Phys. Rev. A 69, 032305 (2004).

[15] C. Santori, D. Fattal, J. Vuckovic, G. S. Solomon, and Y. Yamamoto, Nature (London) 419, 594 (2002).

[16] S. Varoutsis, S. Laurent, P. Kramper, A. Lemaitre, I. Sagnes, I. Robert-Philip, and I. Abram, Phys. Rev. B 72, 041303(R) (2005).

[17] G. Cui and M. G. Raymer, Phys. Rev. A 73, 053807 (2006).
[18] A. Naesby, T. Suhr, P. T. Kristensen, and J. Mork, Phys. Rev. A 78, 045802 (2008).

[19] M. Yamaguchi, T. Asano, and S. Noda, Opt. Express 16, 18067 (2008).

[20] I. Favero, A. Berthelot, G. Cassabois, C. Voisin, C. Delalande, P. Roussignol, R. Ferreira, and J. M. Gerard, Phys. Rev. B 75, 073308 (2007).

[21] A. Berthelot et al. (unpublished).

[22] A. Auffèves, B. Besga, J.-M. Gérard, and J.-P. Poizat, Phys. Rev. A 77, 063833 (2008).

[23] A. Berthelot et al., Nat. Phys. 2, 759 (2006).

[24] R. J. Glauber, in Quantum Optics and Electronics, edited by C. de Witt, A. Blandin, and C. Cohen-Tannoudji (Gordon and Breach, New York, 1965), pp. 65-185.

[25] S. Strauf, K. Hennessy, M. T. Rakher, Y. S. Choi, A. Badolato, L. C. Andreani, E. L. Hu, P. M. Petroff, and D. Bouwmeester, Phys. Rev. Lett. 96, 127404 (2006).

[26] E. Knill, R. Laflamme, and G. J. Milburn, Nature (London) 409, 46 (2001).

[27] S. Reitzenstein, C. Hofmann, A. Gorbunov, M. Strauss, S. H. Kwon, C. Schneider, A. Löffler, S. Höfling, M. Kamp, and A. Forchel, Appl. Phys. Lett. 90, 251109 (2007).

[28] M. Borselli, K. Srivanasan, P. E. Barclay, and O. Painter, Appl. Phys. Lett. 85, 3693 (2004).

[29] E. Weidner, S. Combrie, A. de Rossi, N. V. Q. Tran, and S. Cassette, Appl. Phys. Lett. 90, 101118 (2007).

[30] M. Kaniber, A. Laucht, A. Neumann, J. M. Villas-Boas, M. Bichler, M. C. Amann, and J. J. Finley, Phys. Rev. B 77, 161303(R) (2008).

[31] L. C. Andreani, G. Panzarini, and J. M. Gérard, Phys. Rev. B 60, 13276 (1999). 